Final Technical Report - Nuclear Engineering Education Research

\title{
An Innovative Three-Dimensional Heterogeneous Coarse-Mesh Transport Method for Advanced and Generation IV Reactor Core Analysis and Design
}

\section{DOE Project DE-FG07-05ID14709}

PI: Farzad Rahnema

Nuclear and Radiological Engineering $\backslash$ Medical Physics Programs

George W. Woodruff School

Georgia Institute of Technology

Atlanta, GA 30332

Report Period: (Y1Q1-Y4Q4): 15 Aug 2005 - 14 Aug 2009

\section{Contents:}

1. Summary

2. Project Overview

3. Coarse-Mesh Method

4. New Numerical Benchmark Specifications

5. Coarse-Mesh Method Results

7. References

\section{SUMMARY}

This project has resulted in a highly efficient method that has been shown to provide accurate solutions to a variety of $2 \mathrm{D}$ and $3 \mathrm{D}$ reactor problems. The goal of this project was to develop (1) an accurate and efficient three-dimensional whole-core neutronics method with the following features: based solely on transport theory, does not require the use of cross-section homogenization, contains a highly accurate and self-consistent global flux reconstruction procedure, and is applicable to large, heterogeneous reactor models, and to (2) create new numerical benchmark problems for code crosscomparison. The project was awarded a three-year grant under the NEER program, and was awarded a one-year no-cost extension. The goals of this project have been accomplished.

A three-dimensional Coarse-Mesh Transport Method (COMET) has been developed that uses Monte Carlo simulation at the lattice level, allowing for accurate solutions for fuel assemblies with complex heterogeneity, and without cross-section homogenization. This allows the method to possess the geometric flexibility and accuracy of Monte Carlo methods at the lattice cell level, and the speed and efficiency of conventional nodal methods at the core level. The Coarse-Mesh method has 
been fully developed and tested with both 2D and 3D benchmark problems. In addition, new numerical benchmark problems have been created that are large scale, heterogeneous, and representative of full-scale reactors. These will allow for improved cross-comparison of codes that are designed for whole-core analyses.

\section{PROJECT OVERVIEW}

\section{Task 1.1: Complete the Monte Carlo Adaptation of the 2-D Coarse-Mesh Code}

All goals for this task were accomplished.

MCNP ${ }^{[1]}$ was modified to allow for the inclusion of source sampling from continuous polynomial distributions in space and angle and the expansion of tally routines. This has been fully implemented into a modified version of MCNP as well as the Coarse-Mesh code ${ }^{[2]}$. Convergence of the global eigenvalue of the system was adapted to include an interpolation method which has been fully developed and integrated into the Coarse-Mesh code ${ }^{[3]}$.

The process for generation of response functions has been automated through the generation of batch-file packages. This allows for ease of use in the creation of response functions, as well as the simplification of implementing parallel processing methods for solutions using multi-computer systems. Library interfacing within the Coarse-Mesh code has been modified to standardize formatting through the implementation of NASA's Common Data Format data management package, as well as the FLIB subroutine package developed by Pacific Northwest National Laboratory.

A method for obtaining uncertainties for polynomial expansion routines in MCNP has been developed and implemented into a modification of MCNP. Analysis of the propagation of these errors has been performed, and routines to track the propagation have been fully implemented. Task 1.2: Develop new 2-D benchmark problems

All goals for this task were accomplished.

Benchmark solutions have been developed for two-dimensional BWR problems with zero, forty, and seventy percent void conditions, at hot operating temperatures. Solutions have been developed both with and without the presence of control blades within the BWR using quarter-core configurations. Benchmark solutions have also been developed for 2-D CANDU problems, for a cooled reactor, as well as for checkerboard voided and fully voided conditions, at hot operating conditions.

The lattice depletion code HELIOS ${ }^{[3]}$ was used to obtain accurate multigroup cross-sections and isotopic concentrations for response function generation within MCNP. MCNP was also used to develop a multigroup reference solution for each core at each operating condition.

The generation of a benchmark solution for a 2-D CANDU problem at cold operating conditions was decided to be unimportant for the future development of the Coarse-Mesh code and 
was therefore not developed, in order to move forward with other tasks more in line with the progression of the project. Continuous Energy reference solutions have also been eliminated from the project for the 2-D problems, in favor of moving forward with three dimensional development and other energy treatment methods which are currently under development.

Task 1.3: Test the 2-D Code with new benchmark problems

All goals for this task were accomplished.

The 2-D Coarse-Mesh code was used to solve the reactor problems for the BWR and CANDU configurations and compared against each of the benchmark solution developed for task 1.2. Task 2.1: Modify MCNP Source Code

All goals for this task were accomplished.

The source sampling routine within MCNP has been modified to include sampling from a polynomial source distribution in various variables. In addition, the tally subroutine has been adapted to include tallying on polynomial expansions of variables within MCNP. These modifications have been made to allow for sampling and tallying in three-dimensional problem configurations.

\section{Task 2.2: Develop the 3-D Coarse-Mesh Code}

All goals for this task were accomplished.

The 2-D code has been fully extended to allow for solutions of three-dimensional reactor problems. This new 3-D Code has been fully integrated with all library procedures and automation routines developed during phase one work.

\section{Task 2.3: Benchmark Development in 3-D}

All goals for this task were accomplished.

We have developed several 3-D benchmark solutions for different reactor geometries. A 3D BWR Benchmark problem has been developed based upon data provided by Southern Nuclear, with realistic assembly burn-up and void distributions.

\subsection{Test the 3-D Code using the new Benchmark problems}

All goals for this task were accomplished.

The 3-D Benchmark problems have been modeled with the 3-D Coarse Mesh code, and COMET solutions for the 3-D benchmarks are being prepared for submission to the Annals of Nuclear Engineering Journal (2009).

\subsection{Verify Previous Basis Set (Legendre Polynomials)}

All goals for this task were accomplished.

Legendre Polynomials have been shown to provide very good results for spatial and angle response functions within the coarse-mesh transport method.

$\underline{4.1 \text { Develop and test a whole-core 3D PWR benchmark problem. }}$ 
All goals for this task were met.

A 3D PWR benchmark problem has been developed, and the full description of this benchmark and COMET solutions to it will be published within the next 3 months.

\section{COARSE-MESH METHOD}

\subsection{Overview}

Lets assume that we wish to solve the neutron transport equation for the eigenvalue and angular flux distribution in a large heterogeneous system of volume $V^{[4]}$.

$$
\begin{array}{r}
\hat{\Omega} . \nabla \psi(\vec{r}, \hat{\Omega}, E)+\sigma_{t}(\vec{r}, E) \psi(\vec{r}, \hat{\Omega}, E)-\int_{0}^{\infty} d E^{\prime} \int_{4 \pi} d \Omega^{\prime} \sigma_{s}\left(\vec{r}, \hat{\Omega}^{\prime}, E^{\prime} \rightarrow \hat{\Omega}, E\right) \psi\left(\vec{r}, \hat{\Omega}^{\prime}, E^{\prime}\right) \\
=\frac{1}{4 \pi} \chi(\vec{r}, E) \int_{0}^{\infty} d E \int_{4 \pi} d \Omega \frac{v \sigma_{f}\left(\vec{r}, E^{\prime}\right)}{k} \psi\left(\vec{r}, \hat{\Omega}^{\prime}, E^{\prime}\right)
\end{array}
$$

with arbitrary boundary condition on the surface:

$$
\psi\left(\vec{r}_{b}, \hat{\Omega}, E\right)=B \psi\left(\vec{r}_{b}, \hat{\Omega}^{\prime}, E^{\prime}\right)
$$

where $\hat{n} \cdot \hat{\Omega}<0$ and $\hat{n} \cdot \hat{\Omega}^{\prime}>0, \vec{r}_{b} \in \partial V$. The angular flux at position $\vec{r}$ in the direction $\hat{\Omega}$ and at energy $E$ is represented by $\psi(\vec{r}, \hat{\Omega}, E)$, and system eigenvalue is represented by $k$. The external boundary of the system is denoted by $\partial V$, and the normal vector $\hat{n}$ is chosen as the outward unit vector with respect to $\partial V$, and $B$ is the general boundary condition operator. Solving this equation via direct transport is unrealistic for full scale reactor problems, leading to the development of this method.

The full system is decomposed in a set of $N$ non-overlapping sub-volume elements $V i$ (e.g. coarse mesh), within which, the angular flux distribution can be expressed by the following relation.

$$
\begin{aligned}
\hat{\Omega} . \nabla \psi_{i}(\vec{r}, \hat{\Omega}, E)+\sigma_{t}(\vec{r}, E) \psi_{i}(\vec{r}, \hat{\Omega}, E)-\int_{0}^{\infty} d E^{\prime} \int_{4 \pi} d \Omega^{\prime} \sigma_{s}\left(\vec{r}, \hat{\Omega}^{\prime}, E^{\prime} \rightarrow \hat{\Omega}, E\right) \psi_{i}\left(\vec{r}, \hat{\Omega}^{\prime}, E^{\prime}\right) \\
=\frac{1}{4 \pi} \chi(\vec{r}, E) \int_{0}^{\infty} d E \int_{4 \pi} d \Omega \frac{v \sigma_{f}\left(\vec{r}, E^{\prime}\right)}{k} \psi_{i}\left(\vec{r}, \hat{\Omega}^{\prime}, E^{\prime}\right)+Q_{i}(\vec{r}, \hat{\Omega}, E)
\end{aligned}
$$

with boundary condition:

$\psi_{i}^{-}\left(\vec{r}_{i j}, \hat{\Omega}, E\right)=\psi_{j}^{+}\left(\vec{r}_{i j}, \hat{\Omega}, E\right)$ where $\vec{r}_{i j} \in\left\{V_{i} \cap V_{j}\right\}$ for all $V_{i}$ bounding $V_{j}$

In this formulation, $k$ is still the system eigenvalue (not the cell), $\psi_{i}$ represents the angular flux within the volume element $i$ and $V j$ represents all the sub-volume elements sharing a common boundary with $V i$. The "+" and "-" signs of the angular flux indicate the outgoing and incoming 
direction. For the particular case where the volume element shares a boundary with the external boundary of the system, the boundary condition of the volume element takes the form:

$$
\psi_{i}^{-}\left(\vec{r}_{i b}, \hat{\Omega}_{i}, E\right)=B \psi_{i}^{+}\left(\vec{r}_{i b}, \hat{\Omega}_{i}{ }^{\prime}, E^{\prime}\right) \text { where } \vec{r}_{i b} \in\left\{V_{i} \cap \partial V\right\}
$$

Applying this decomposition of the heterogeneous system leads to a series of $N$ fixed source problems, as shown in equation (3), which are entirely equivalent to solving the problem of equation (1). For eigenvalue calculations, this leads to a two-level iterative process. The outer iterations are performed on the eigenvalue, while the inner iterations are performed on the surface angular fluxes or any other quantity of interest (e.g. fuel pin fission density or local dose distribution in the case of shielding or medical physics problems). The implicit treatment of the fission and scattering sources within the fixed source problem leads to a significant improvement in overall efficiency over similar methods that require both incident flux and in-volume source responses. In addition, this formulation makes no approximation regarding the shape of the fission source distribution, which is necessary with methods that employ in-volume responses. However, with the implicit source treatment, the magnitude of the source is controlled by the incident flux in the local fixed source calculation, thus needing an external normalization in order to converge on the global system solution.

\subsection{Approximation}

The method described in the previous section is exact but it is based on the assumption that the surface flux distribution in angle, space and energy of each coarse mesh is known. To resolve this, an approximation is introduced. The local fixed source problem of equation (3) is solved with the following boundary condition:

$$
\psi_{i}^{-}\left(\vec{r}_{i s}, \hat{\Omega}, E\right)=\left\{\begin{array}{cc}
\Gamma^{m}\left(\vec{r}_{i s}, \hat{\Omega}, E\right) & \text { where } \vec{r}_{i s} \in\left\{V_{i} \cap \partial V_{s}\right\} \\
0 & \text { elsewhere }
\end{array}\right.
$$

where $\partial V_{s}$ denotes a sub-region of the boundary of coarse-mesh $V j$ that shares a boundary with $V i$, and $\Gamma^{m}\left(\vec{r}_{i s}, \hat{\Omega}, E\right)$ is the $m t h$ member of a set of functions that are orthogonal on the infinitedimensional half-space. The solution of the fixed source problem with that boundary condition

provides a response function denoted $R_{i s}^{m}\left(\vec{r}_{i s}, \hat{\Omega}, E\right)$. This response function is the actual angular flux solution corresponding to a given boundary condition $\Gamma^{m}\left(\vec{r}_{i s}, \hat{\Omega}, E\right)$, it thus gives the response of the coarse mesh to this particular boundary condition. Then the solution to the local fixed source problem within coarse- mesh $V i$ can be constructed as the linear superposition:

$$
\psi_{i}(\vec{r}, \hat{\Omega}, E)=\sum_{m=0}^{\infty} \sum_{s} c_{i s}^{m} R_{i s}^{m}(\vec{r}, \hat{\Omega}, E)
$$


with coefficients $c_{i s}^{m}$ determined by:

$$
c_{i s}^{m}=\iiint \psi_{i}^{-}\left(\vec{r}_{i s}, \hat{\Omega}, E\right) \Gamma^{m} d \vec{r}_{i s} d \hat{\Omega} d E
$$

For computational efficiency, it is desirable to truncate the response expansion at a low order. This is the only approximation in the coarse mesh method. The accuracy obtained for a given maximum order depends, of course, on the type of response functions used.The accuracy of this approximation depends on the order of the calculation and what type of response functions used. It was found in this project that very good accuracy can be obtained with the use of Legendre Polynomials as the basis of the response functions.

\subsection{Monte Carlo Response Function Adaptation}

The decision to pursue the development of a Monte Carlo adaptation is due to many factors in addition to its accuracy. These include 1) the geometric flexibility, 2) the possibility of doing both multi-group and continuous-energy calculations, 3) the use of continuous orthogonal expansions, 4) wide use and availability of the method... It should be noted that the coarse mesh deterministic method is totally independent of the technique/code used to generate the response functions and that the accuracy of the global solution is limited by the accuracy of the fine-mesh code. While MCNP is not widely regarded as the most efficient tool for performing pre-computations, it is our experience that for small fixed source calculations with vacuum boundary conditions, MCNP is as efficient as most publicly available deterministic codes ${ }^{[4]}$.

Generating Response functions with a Monte Carlo code requires several adaptations of ordinary Monte Carlo techniques, and thus MCNP was modified to allow for the inclusion of source sampling from continuous polynomial distributions in space and angle and the expansion of tally routines to generate response functions. This has been fully implemented into a modified version of MCNP as well as the Coarse-Mesh code ${ }^{[2]}$.

The distribution of particles along a given surface is defined by a tensor product of Legendre polynomials orthogonal over given intervals specified by their respective variables, which leads the boundary condition of equation (6) to be of the form:

$$
\Gamma_{g}^{m, n, p, q}(x, y, \mu, \varphi, E)=\delta_{g} P_{m}(u) P_{n}(v) P_{p}(\varphi) P_{q}(\mu)
$$

where $x$ and $y$ are the spatial variables, $\mu$ is the polar angle and $\varphi$ is the azimuthal angle. The indices $m, n, p$ and $q$ are the order of expansion of the corresponding variables. Sampling each variable within the modified MCNP for orders $m, n, p$ and $r$ will determine the location, direction and velocity of the particle emerging from the given surface. For multigroup calculations, the energy variable is treated like a delta function for a given energy group $g$. 


\subsection{Implementation}

\subsubsection{Eigenvalue Interpolation}

In early work on the Coarse-Mesh method [5], the eigenvalue was evaluated as a ratio of gains to losses according to the following formula:

$$
k=\frac{\iiint F \psi d \vec{r} d \hat{\Omega} d E}{\iiint A \psi d \vec{r} d \hat{\Omega} d E+L}
$$

where $F$ is the usual fission operator, $A$ is the absorption operator and $L$ is the net leakage out of the system.

The new method, referred to as the normalization method (NM) is based on the knowledge that the normalization constant $(C)$ will be equal to unity once outer convergence is achieved. In an attempt to accelerate convergence, the normalization constant is evaluated at two distinct guesses of $k$, and they are found to be related by the following equation:

$$
C_{i, 1}-C_{i, 2} \propto v \sigma_{f}\left(\frac{1}{k_{1}}-\frac{1}{k_{2}}\right)\left(\int \phi_{i, 1}(\vec{r}) \phi_{i, 2}(\vec{r}) d V\right)
$$

where $C i, 1$ and $C i, 2$ are the normalization constants for eigenvalues $k 1$ and $k 2$ within coarse mesh $i$, and $\phi$ is the scalar flux. This information is used to linearly interpolate the value of $k$ for which the normalization constant will be equal to unity ${ }^{[6]}$.

\subsubsection{Automation of Response Function Generation}

Modeling of a complicated reactor system can require thousands of response functions to be generated, and within the Monte Carlo adaptation, this requires thousands of fixed-source MCNP calculations to be run, with results stored and accessible. Creating and running so many MCNP input files by hand would be extremely time-consuming. This has been significantly reduced in the COMET implementation by the use of system-dependent batch files. A set of MCNP templates is created by the user that describes each geometrically and materially unique coarse mesh type (typically less than 20 types for a reactor model), and the coarse mesh code automatically generates response function input files into a user-specified number of folders, as well as a batch file for each folder to run all the response function calculations it contains, and rename the tally files to keep track of them. This allows the MCNP calculations to be divided up among several computers and run in parallel, greatly reducing the response function generation time. The resulting MCNP Tally files are then used by the coarse mesh code to generate a response function database.

\subsubsection{External Libraries}

Two external libraries were used within the coarse mesh code transport method to allow for efficient handling of the large number of response functions generated with the method. The PNNL 
Fortran Library (a.k.a. FLIB) is a set of general purpose Fortran 90 routines that can be used to simplify the development of programs with a batch-style input/output interface. This library was used primarily to efficiently handle the input and output requirements with the large number of files generated in the coarse-mesh code. In addition, the response function database requires a significant amount of memory handling, and the development of the coarse mesh code utilizes the Common Data Format Library developed by NASA/Goddard Space Flight Center. This library is used for the creation and accessing of a central response function database more efficiently than through standard file access.

\section{NEW NUMERICAL BENCHMARK SPECIFICATIONS}

A significant gap in the knowledge base of the Nuclear Engineering industry has been the lack of whole core benchmark problems. As a part of this project, the following benchmark problems were prepared. Full Benchmark suites including 1D, 2D, and 3D BWR and PWR benchmarks are completed, and specifications and COMET solutions to them are being submitted for publication in the next 3 months in peer-reviewed journals and conference proceedings as a result of this project.

\subsection{Two-Dimensional Benchmark Specifications}

\subsubsection{D Boiling Water Reactor Benchmark Specification ${ }^{[7]}$}

The BWR benchmark consists of a checkerboard layout of fresh ( $0 \mathrm{GWd} / \mathrm{TU})$ and depleted (17 GWd/TU) GE9 assemblies ${ }^{[8]}$. The core is composed of 308 square fuel assemblies of length 15.24 $\mathrm{cm}$, surrounded by assemblies of moderator, and then vacuum. The problem is simplified via implementing $1 / 8^{\text {th }}$ symmetry, laid out as in Figure 1. Due to the presence of control blades in several assemblies, there are 8 distinct assembly types in the BWR problem:

1. Fresh, 00\% Voiding (F00)

2. Fresh, $40 \%$ Voiding (F40)

3. Fresh, 70\% Voiding (F70)

4. Fresh, Controlled (FC)

5. Depleted, $00 \%$ Voiding (D00)

6. Depleted, $40 \%$ Voiding (D40)

7. Depleted, $70 \%$ Voiding (D70)

8. Depleted, Controlled (DC) 


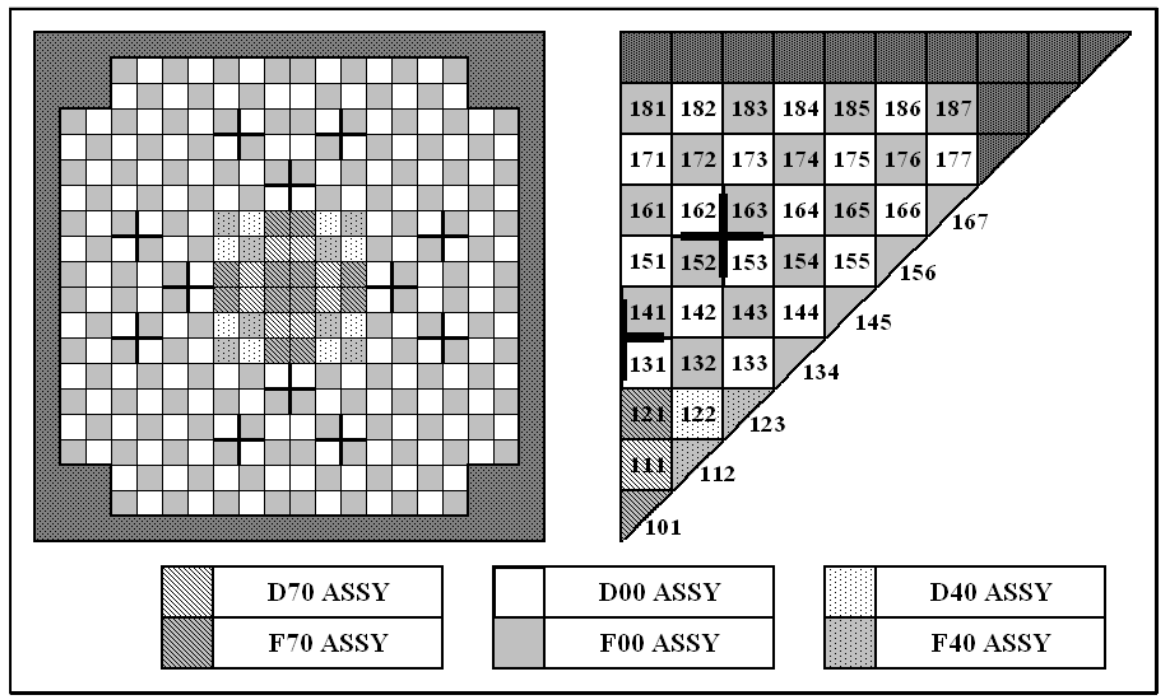

Figure 1: BWR Core Layout

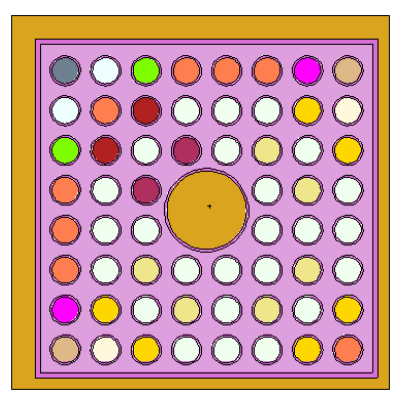

(a)

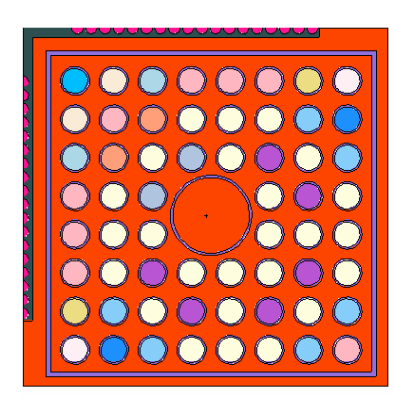

(b)

Figure 2: BWR Assembly Layout for an (a) uncontrolled case and a (b) controlled case.

The assemblies were modeled using $1 / 8^{\text {th }}$ symmetry and specular reflective boundary conditions on all sides, and the lattice depletion code HELIOS was used to generate few-group crosssections. The BWR core was run on a 96-core computer cluster with 1 billion neutron histories, using the multi-group option and the generated cross section library. Nominally 250,000 particles were simulated in each cycle, with 4000 cycles run (200 inactive for source convergence). The reference eigenvalue of this core was $0.99489 \pm 0.00002$, with fission densities tallied in all pins.

\subsubsection{D Pressurized Water Reactor Benchmark Specification ${ }^{[7]}$}

The PWR benchmark consists of two different assembly designs: Uranium Oxide (UO2) and Mixed Oxide (MOX), laid out in grid, as in figure 3. The design of the fuel assemblies is from the C5G7 fuel assemblies used in NEA MOX benchmark ${ }^{[9]}$. The UO2 assembly is composed of only one type of fuel, and the MOX assembly is composed of three different enrichments of transuranic isotopes. The moderator is chosen to be a fictional moderator of low density to generate higher 
anisotropy in the flux, and challenge reactor simulators. Control is maintained in the reactor by means of boron control rods which are inserted into 24 guide tubes in the fuel assemblies.

The core is composed of 121 square fuel assemblies of length $21.42 \mathrm{~cm}$, surrounded by assemblies of moderator, and then vacuum. Due to the presence of control rods in several assemblies, there are 5 distinct assembly types in the PWR problem:

1. Uncontrolled UO2 (UO2)

2. Controlled UO2 (UO2-C)

3. Uncontrolled MOX (MOX)

4. Controlled MOX (MOX-C)

5. Moderator (CLT)

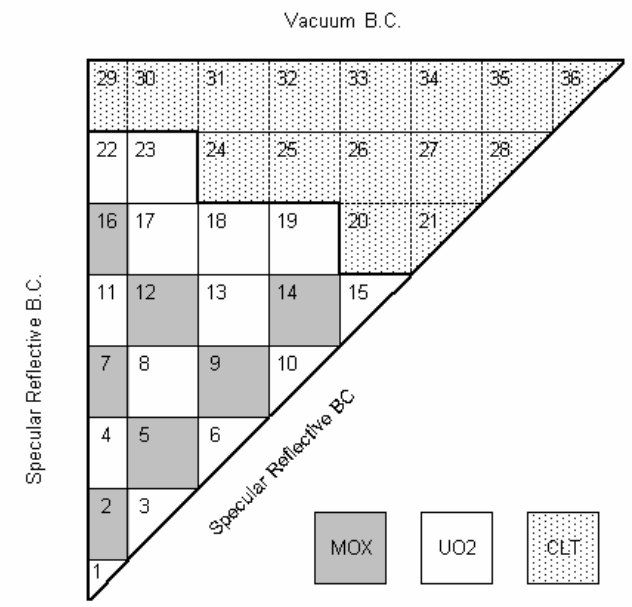

Figure 3. $1 / 8^{\text {th }}$ Symmetry PWR Layout with Assembly Indices

Each assembly consists of a $17 \times 17$ square lattice of pin cells, with 24 guide tubes/control rods evenly spaced throughout, and one central guide tube, as in Figure 4. Centered in the pin cell is a circular pin of fuel, surrounded by zirconium cladding.

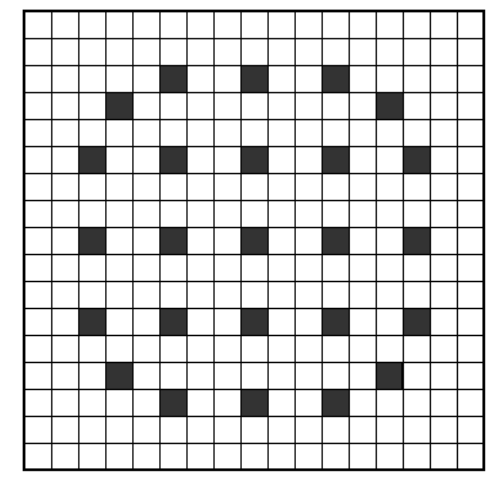

Guide Tube / Control Rod UO2 Pin

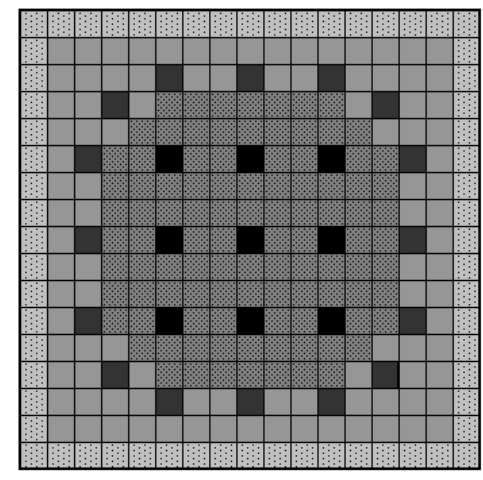

Guide Tube / Control Rod MoX8.7 Pin MOX7.0 Pin MOX43 Pin 
Figure 4: PWR Fuel Assemblies (UO2 Left, MOX Right)

The assemblies were modeled using $1 / 8^{\text {th }}$ symmetry and specular reflective boundary conditions on all sides, and the lattice depletion code HELIOS was used to generate few-group crosssections. The PWR core was run on a 160-core computer cluster with 1 billion neutron histories, using the multi-group option and the generated cross section library. Nominally 250,000 particles were simulated in each cycle, with 4000 cycles run (200 inactive for source convergence). The reference eigenvalue of this core was $1.00294 \pm 0.00002$, with fission densities tallied in all pins. 4.1.3 2D CANDU Reactor Benchmark Specification ${ }^{[10]}$

The 2-D quarter core CANDU-6 benchmark problem is representative of a simplified upper right hand corner of an operating CANDU-6 reactor as shown in Figure 5 .

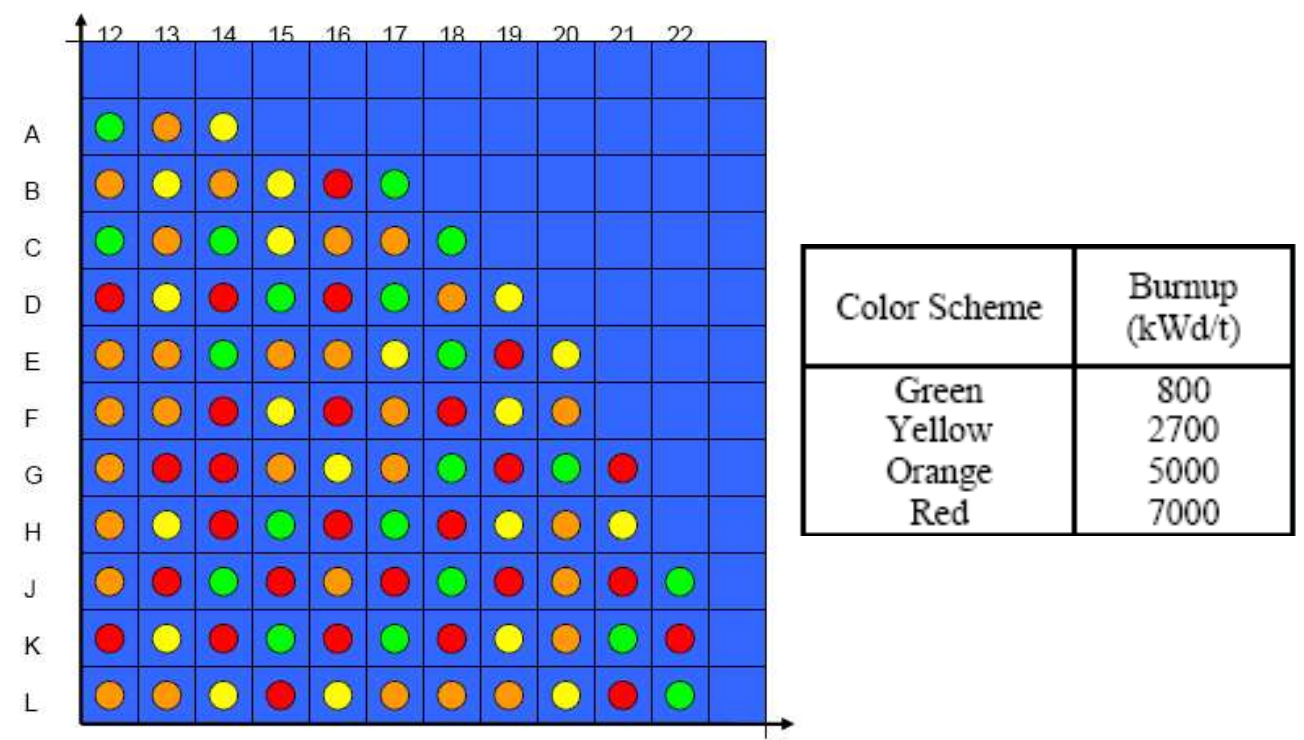

Figure 5. CANDU-6 Core Layout

The 95 fuel assemblies are exact representations of 37 fuel elements CANDU-6 bundles shown in Figure 6.

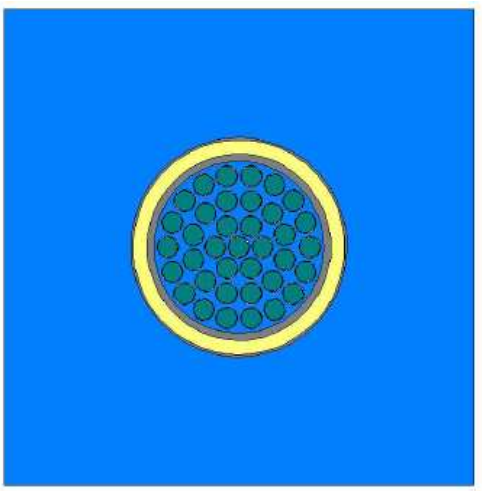


Figure 6. CANDU-6 37-element Fuel Bundle

Reflective boundary conditions are used on the west and south surfaces and vacuum elsewhere. Four different burnups $(800 \mathrm{kWd} / \mathrm{t}, 2700 \mathrm{kWd} / \mathrm{t}, 5000 \mathrm{kWd} / \mathrm{t}, 7000 \mathrm{kWd} / \mathrm{t})$ were chosen and distributed according to an operating core burnup map as illustrated by Figure 7. Depletion calculations were performed on a single bundle infinite lattice calculation using the lattice depletion code HELIOS from which two group cross-sections were obtained. All fuel elements are represented by a single set of cross-sections for each burnup. A separate set of cross-sections is also used for the moderator, the coolant and the cladding. The gap normally filled with gas was considered to be a void in the HELIOS calculation. A two group MCNP reference solution was obtained using 2.1 billion active particles on a previously well converged source. The core has an eigenvalue of $1.01943 \pm$ 0.00001 with average and maximum pin power uncertainties of $0.06 \%$ and $0.10 \%$, respectively.

\subsection{Three-Dimensional Benchmark Specifications}

\subsubsection{D Pressurized Water Reactor Benchmark Extension ${ }^{[11]}$}

The three dimensional PWR specification is an axially extended version of the 2-dimensional specification above. It includes the same assembly types, plus a tube/spring assembly and plug assembly for the top and bottom of the axial layout in Figure 7 with vacuum conditions on all sides.

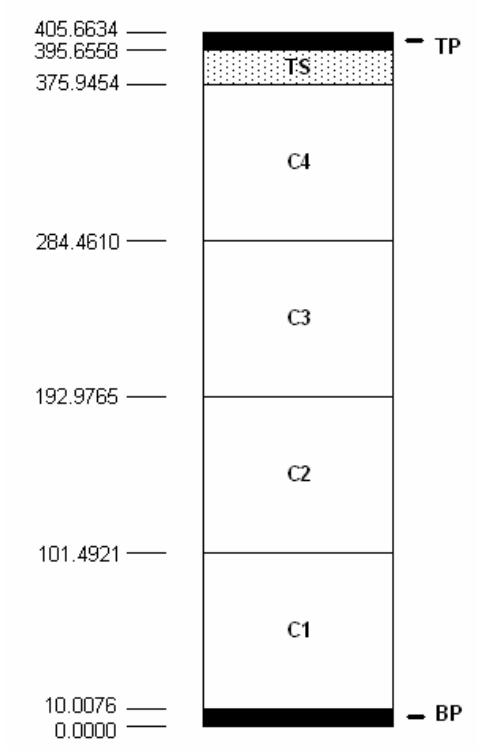

Figure 7. 3D PWR Axial Layout

The Tube/Spring axial layer is composed of geometry identical to the fuel assemblies, where the fuel material has been replaced with zirconium cladding material. The other materials in the controlled and uncontrolled tube/spring assemblies are the same materials as the controlled and uncontrolled $\mathrm{UO}_{2}$ assemblies, respectively. The plug assembly materials are the same, but the guide tube regions are also replaced with zirconium cladding material. In addition, the moderator from the 
2D assembly was modified to be a realistic moderator and includes $1800 \mathrm{ppm}$ natural Boron as a burnable poison. With the addition of these assembly types, there are now 7 different unique assemblies, as in table I.

Table I: PWR Assembly Types

Assembl
Number
1
2
3
4
5
6
7

$$
\begin{gathered}
\text { Assembly } \\
\text { Type } \\
\mathrm{UO}_{2}-\text { Controlled } \\
\mathrm{UO}_{2}-\text { Uncontrolled } \\
\mathrm{MOX}-\text { Controlled } \\
\mathrm{MOX} \text { - Uncontrolled } \\
\text { Tube / Spring - Uncontrolled } \\
\text { Tube / Spring - Controlled } \\
\text { Plug }
\end{gathered}
$$

\begin{tabular}{|c|c|c|c|c|c|c|c|c|c|c|c|c|c|c|c|c|c|c|c|c|c|}
\hline $\mathbf{S U}$ & - & $\sim$ & $m$ & $\nabla$ & in & 6 & $r$ & $\infty$ & $a$ & $\stackrel{0}{ }$ & $=$ & $\simeq$ & 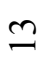 & $\Xi$ & $n$ & $\underset{0}{0}$ & I & 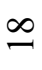 & 9 & ป & $\tilde{\sim}$ \\
\hline TP & 7 & 7 & 7 & 7 & 7 & 7 & 7 & 7 & 7 & 7 & 7 & 7 & 7 & 7 & 7 & 7 & 7 & 7 & 7 & 7 & 7 \\
\hline TS & 6 & 5 & 6 & 5 & 5 & 5 & 5 & 5 & 5 & 6 & 6 & 5 & 6 & 5 & 5 & 5 & 6 & 5 & 5 & 5 & 5 \\
\hline $\mathrm{C} 4$ & 2 & 4 & 2 & 2 & 4 & 2 & 4 & 2 & 4 & 2 & 1 & 4 & 2 & 4 & 2 & 4 & 2 & 4 & 2 & 2 & 2 \\
\hline $\mathrm{C} 3$ & 2 & 4 & 2 & 1 & 4 & 2 & 4 & 2 & 4 & 2 & 1 & 4 & 2 & 4 & 2 & 4 & 2 & 4 & 2 & 2 & 2 \\
\hline $\mathrm{C} 2$ & 2 & 4 & 2 & 1 & 4 & 2 & 4 & 2 & 4 & 2 & 2 & 4 & 2 & 4 & 2 & 4 & 2 & 4 & 2 & 2 & 2 \\
\hline $\mathrm{C} 1$ & 2 & 4 & 2 & 1 & 4 & 2 & 4 & 2 & 4 & 2 & 2 & 4 & 2 & 4 & 2 & 4 & 2 & 4 & 2 & 2 & 2 \\
\hline BP & 7 & 7 & 7 & 7 & 7 & 7 & 7 & 7 & 7 & 7 & 7 & 7 & 7 & 7 & 7 & 7 & 7 & 7 & 7 & 7 & 7 \\
\hline
\end{tabular}

The full-power operating condition of the reactor is selected as a configuration with one control bank halfway inserted and the partial length rod fully inserted, as in figure 8 .

Figure 8. Full-Power Operational Configuration by assembly type: Assembly Indices ( Horizontally, and Axial Layer Vertically. Controlled Regions are shaded.

The 3D PWR core was run on a 160-core computer cluster with 1 billion neutron histories, using the multi-group option and the generated cross section library. (Same as in the 2D model) Nominally 250,000 particles were simulated in each cycle, with 4000 cycles run (200 inactive for source convergence). The reference eigenvalue of this core was $1.00357 \pm 0.00003$, with fission densities tallied in all pins. The published version of this benchmark (submitted 11/2009) includes fully rodded and unrodded configurations and pin fission densities.

\subsubsection{D Boiling Water Reactor Benchmark Extension ${ }^{[12]}$}

The three dimensional BWR specification is an axially extended version of the 2-dimensional specification above with an overall length of $381.0 \mathrm{~cm}$. It includes the same types of assembly, extended axially into 5 equal height zones, but with an axial void distribution based on the HATCH-II 
reactor and burnup parameters in the core. The HATCH-II benchmark problem is not the same overall geometry as the HAFAS-based benchmark presented here, and the void distribution is modified to remain as close to the physics represented in the HATCH-II model.

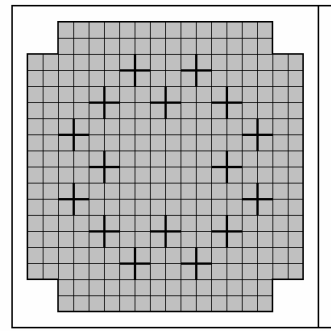

(a)

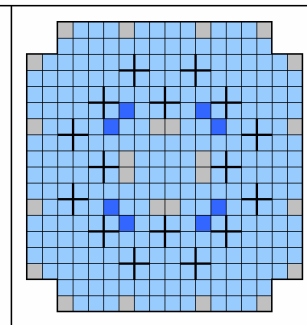

(b)

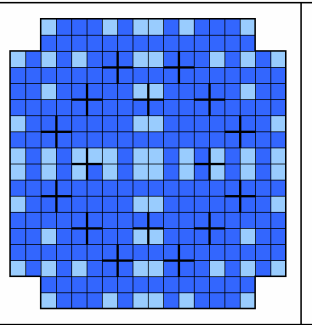

(c)

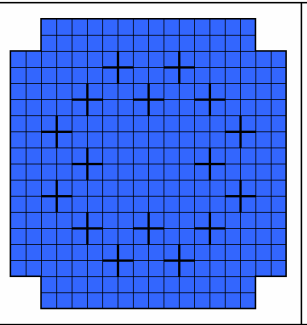

(d)

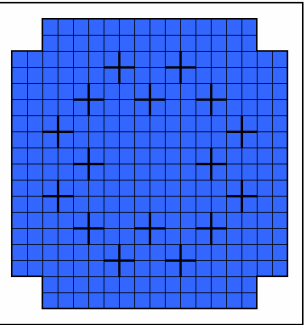

(e)

Figure 9. 3D BWR Axial Void Layout for each layer (a) bottom to (e) top.

Grey (0\%), Light Blue (40\%), and Dark Blue (70\%)

Reactivity control is maintained in the core by control rod blades in between assemblies (shown in figure 9). As in the case of the 3D PWR, the full publication of this benchmark specification (submitted 11/2009), includes additional rod configurations and pin fission densities.

The 3D BWR core was run on a 160-core computer cluster with 1 billion neutron histories, using the multi-group option and the generated cross section library. (Same as in the 2D model) Nominally 250,000 particles were simulated in each cycle, with 4000 cycles run (200 inactive for source convergence). The reference eigenvalue of this core was $1.00555 \pm 0.00002$, with fission densities tallied in all pins.

\section{COARSE-MESH METHOD RESULTS}

\subsection{D CANDU-6 Quarter Core Benchmark Problem}

The Coarse-Mesh Transport Method was applied to the Quarter Core Benchmark Problem described earlier ${ }^{[10]}$. Low-order response functions were computed using the modified version of MCNP. Each response function was computed using 3 million particles which took about 6 minutes for fuel assemblies and 1 minute for moderator assemblies. The pin powers convergence criterion was set to $10^{-4}$. The results of the coarse mesh method are shown in Table II.

Table II. Coarse Mesh Results - CANDU-6 Quarter Core

\begin{tabular}{|c|c|c|c|}
\hline & $\begin{array}{c}\{0,0,0\} \\
(\%)\end{array}$ & $\begin{array}{c}\{1,0,0\} \\
(\%)\end{array}$ & $\begin{array}{c}\{2,2,2\} \\
(\%)\end{array}$ \\
\hline$R E k$ & -0.60 & 0.41 & 0.09 \\
\hline$A V G$ & 3.0 & 0.5 & 0.5 \\
\hline$R M S$ & 3.6 & 0.7 & 0.6 \\
\hline$M R E$ & 2.8 & 0.5 & 0.4 \\
\hline$M A X$ & 9.2 & 2.4 & 2.0 \\
\hline CPU time (s) & 2.0 & 2.9 & 31.7 \\
\hline
\end{tabular}




\subsection{D BWR Quarter Core Benchmark Problem}

The Coarse-Mesh Transport Method was applied to a 2D BWR Quarter Core Benchmark Problem similar to the one described earlier, with changes in some geometric parameters (assembly pitch and control blade description) ${ }^{[13]}$. The reference core solution in half of the problem (diagonal symmetry) is obtained by using 230,000 particles per cycle over 6,000 active cycles. The fission source for this simulation was obtained by skipping 500 cycles. This calculation took approximately 60 hours on a 15-node cluster of dual-core Pentium D 3.0GHz computers running the Windows operating system. The reference core eigenvalue is $1.03592 \pm 0.00002$. Fission densities in all fuel pins are tallied. The average and maximum fission density relative errors (uncertainty) are $0.09 \%$ and $0.18 \%$, respectively.

Each simulation was performed using 5 million active particles. Each calculation takes approximately 15 minutes in the fuel mesh and 5 minutes in the reflector mesh on a $2.8 \mathrm{GHz}$ Pentium 4 PC, respectively. Using the same 15-node cluster mentioned previously, the total RF precomputation time took approximately 50 hours

Table III: Coarse-Mesh Results in the BWR Benchmark Problem

\begin{tabular}{|c|c|c|c|c|}
\hline & $\begin{array}{c}\{2,2,2\} \\
(\%)\end{array}$ & $\begin{array}{c}\{3,2,2\} \\
(\%)\end{array}$ & $\begin{array}{c}\{3,2,3\} \\
(\%)\end{array}$ & $\begin{array}{c}\{4,2,3\} \\
(\%)\end{array}$ \\
\hline RE $k$ & -0.21 & -0.12 & -0.13 & -0.13 \\
\hline AVG & 3.0 & 2.0 & 1.8 & 1.7 \\
\hline RMS & 3.5 & 2.3 & 2.1 & 2.0 \\
\hline MAX & 10.3 & 7.4 & 7.1 & 6.5 \\
\hline CPU time (s) & 151 & 238 & 394 & 593 \\
\hline
\end{tabular}

$\{a, b, c\}: a^{\text {th }}$ order expansion in space

$\mathrm{b}^{\text {th }}$ order expansion in cos (polar angle)

$\mathrm{c}^{\text {th }}$ order expansion in azimuthal angle

\subsection{D PWR Whole-Core Benchmark Problem}

A PWR full core was constructed using the uranium oxide (UO2) and mixed oxide fuel assemblies (MOX) of the C5G7 problem ${ }^{[14]}$. The core is composed of 48 uncontrolled UO2 assemblies, 21 controlled UO2 assemblies, 28 uncontrolled MOX assemblies and 24 controlled MOX assemblies. The core geometry is presented in Figure 10. 


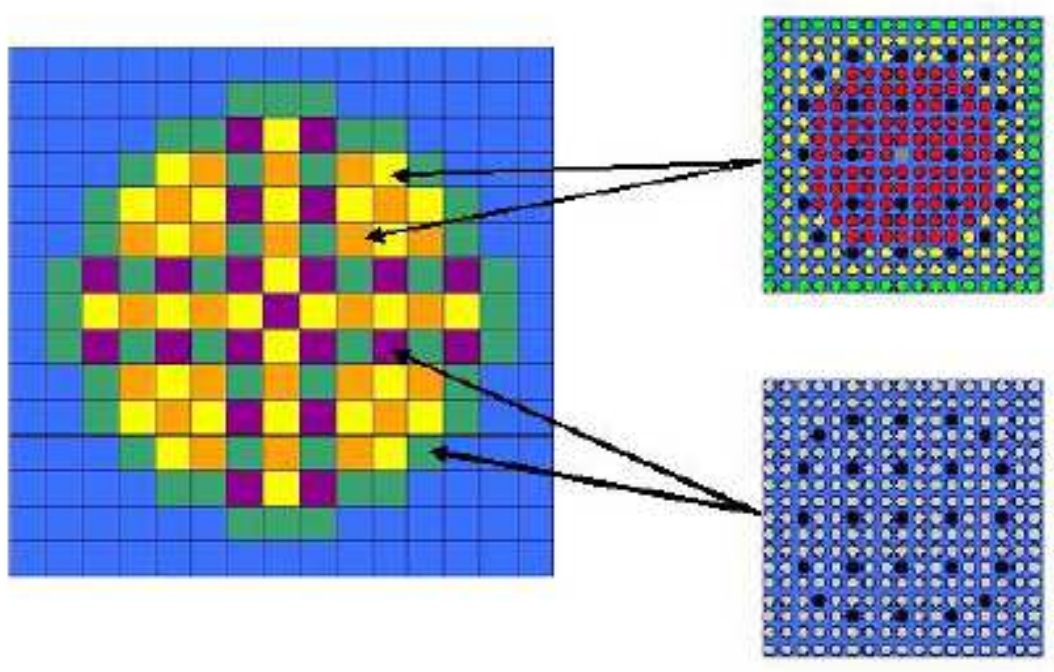

Figure 10. Whole-Core 2D Benchmark Problem

In this case, the 7-group cross section library from the $\mathrm{C} 5 \mathrm{G} 7$ problem was used to perform the reference MCNP and COMET calculations. The reference solution was obtained by performing MCNP5 calculation in 1/8th of the core using 1.6 billion active particles with a converge source. The reference eigenvalue of the core is $1.12623 \pm 0.00002$. The average pin power uncertainty is $0.11 \%$ with a maximum uncertainty of $0.23 \%$. The coarse-mesh result is presented in table IV.

Table IV. 2D Whole-Core PWR Coarse-Mesh Solution

\begin{tabular}{|c|c|c|}
\hline & $\begin{array}{c}\left\{2^{2}, 2,2\right\} \\
(\%)\end{array}$ & $\begin{array}{c}\left\{3^{2}, 2,4\right\} \\
(\%)\end{array}$ \\
\hline RE $k$ & -0.01 & -0.01 \\
\hline $\mathrm{AVG}$ & 0.5 & 0.4 \\
\hline RMS & 0.4 & 0.4 \\
\hline MAX & 4.1 & 1.7 \\
\hline CPU time (s) & 23.0 & 112.3 \\
\hline
\end{tabular}

\subsection{D C5G7 MOX Benchmark Problem}

In the extended 3-D OECD/NEA Benchmark configurations ${ }^{[9]}$, the fuel assemblies are surrounded on three sides by a reflector region with vacuum boundary conditions, while the other three sides have specular reflective boundary conditions. The core height was $64.26 \mathrm{~cm}$. The presence of control rods is also added as an additional element of complexity for the problems, as in figures $11-13$. 

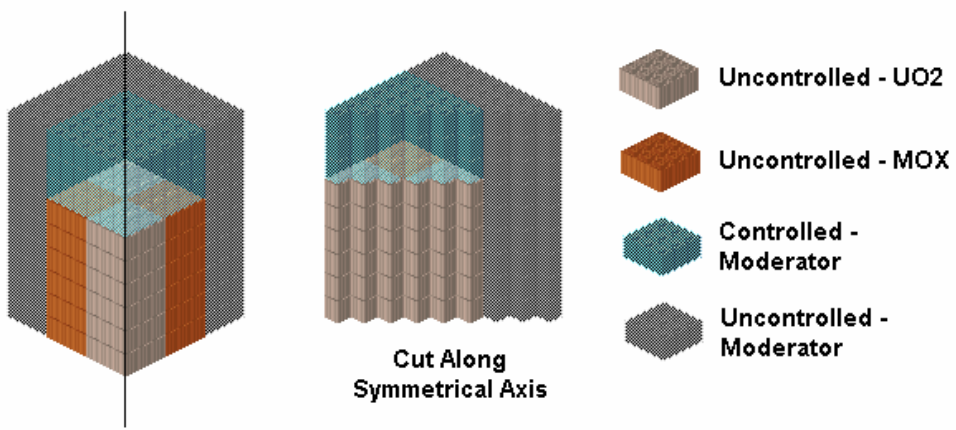

Figure 11. Unrodded C5G7 Configuration
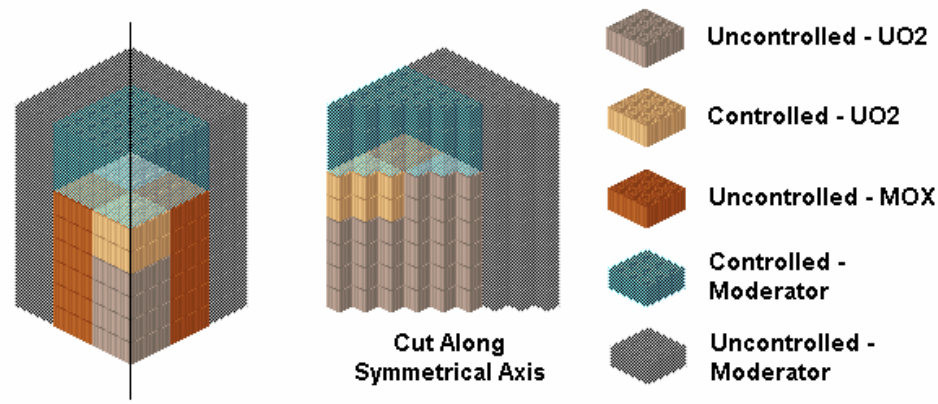

Figure 12. Rodded A C5G7 Configuration
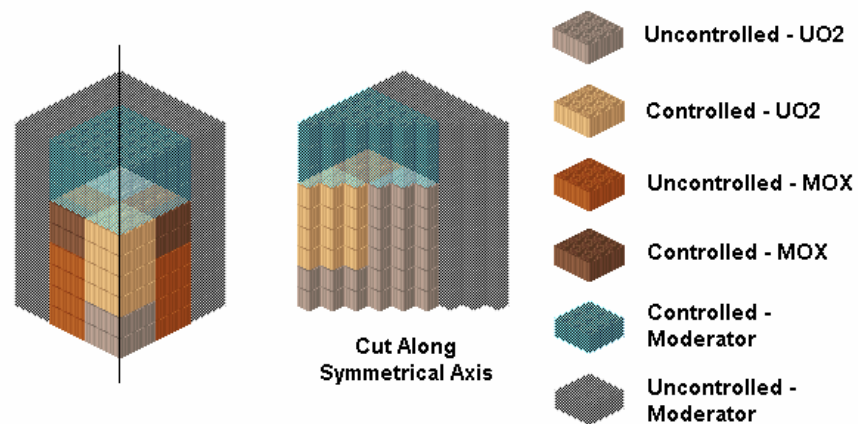

Figure 13. Rodded B C5G7 Configuration

Coarse mesh solutions were obtained for these geometries ${ }^{[15]}$, and in all calculations, the convergence criteria for the eigenvalue and the pin powers are both $10^{-4}$. The eigenvalue relative error and the computational time required for the 2 nd order solution of each of these configurations can be found in Table $\mathrm{V}$. It should be noted that the computational time indicated in the aforementioned table is only indicative of the time needed to perform the coarse mesh calculation, which was performed on a single $2.8 \mathrm{GHz}$ Intel Pentium 4 processor. In all three cases, the eigenvalue relative error is small, within three standard deviations of the calculational uncertainties. Table VI shows a summary of the pin power error distributions for all configurations. 
Table V. Coarse Mesh Solution for 3D C5G7 Benchmark Problem

\begin{tabular}{|c|c|c|c|}
\hline & Unrodded & Rodded A & Rodded B \\
\hline Reference Eigenvalue & $1.14308 \pm 0.00007$ & $1.12806 \pm 0.00007$ & $1.07777 \pm 0.00006$ \\
\hline COMET Eigenvalue & $1.1437 \pm 0.0002$ & $1.1287 \pm 0.0002$ & $1.0784 \pm 0.0002$ \\
\hline Eigenvalue RE (\%) & $0.06 \pm 0.02^{\mathrm{a}}$ & $0.06 \pm 0.02^{\mathrm{a}}$ & $0.06 \pm 0.02^{\mathrm{a}}$ \\
\hline CPU time (s) & 820 & 832 & 836 \\
\hline
\end{tabular}

a. uncertainty of the relative error $\sqrt{\left(\partial R E / \partial k_{\text {COMET }}\right)^{2} S_{C O M E T}^{2}+\left(\partial R E / \partial k_{M C N P}\right)^{2} S_{M C N P}^{2}}$

The unrodded configuration has very little axial heterogeneity. The control rods are only present in the axial reflector nodes and the core is essentially a 2-D problem. As expected, the results for this case are good even with such a low-order (2nd) spatial and angular approximations. The Rodded $A$ configuration adds an element of complexity due to the presence of the control rods in the upper lefthand corner of the radial plane at $14.28 \mathrm{~cm}$. The control rods are thus fully inserted in that assembly in slice 3 . The Rodded $B$ configuration exhibits an even greater axial heterogeneity caused by the insertion of the upper left hand control rod bank to $28.56 \mathrm{~cm}$ and the insertion of the lower lefthand control rod bank and the upper right-hand control rod bank to $14.28 \mathrm{~cm}$. Results for the Coarsemesh method are on the same order for all configurations of the benchmark problem, and are good for even a low-order (2nd) spatial and angular approximation. It has been shown ${ }^{[13]}$ that by extending the expansion to a higher order in space, the maximum pin power error can be reduced significantly, as shown in table VI as well.

Table VI. Pin Power Summary for Coarse-Mesh Solution to 3D C5G7 Benchmark

\begin{tabular}{|c|c|c|c|c|}
\hline \multicolumn{5}{|c|}{ Unrodded } \\
\hline & $\mathrm{AVG}$ & RMS & MRE & MAX \\
\hline Slice $1\{2,2,2,2\}$ & 1.0 & 1.5 & 0.8 & 9.4 \\
\hline Slice $2\{2,2,2,2\}$ & 1.0 & 1.5 & 0.7 & 9.9 \\
\hline Slice $3\{2,2,2,2\}$ & 1.2 & 1.7 & 0.9 & 10.3 \\
\hline Total $\quad\{2,2,2,2\}$ & 1.0 & 1.5 & 0.7 & 9.8 \\
\hline Total $\quad\{3,3,2,2\}$ & 0.7 & 1.1 & 0.6 & 9.7 \\
\hline Total $\{4,4,2,2\}$ & 0.5 & 0.7 & 0.4 & 3.3 \\
\hline \multicolumn{5}{|c|}{ Rodded $A$} \\
\hline & AVG & RMS & MRE & MAX \\
\hline Slice $1\{2,2,2,2\}$ & 1.0 & 1.5 & 0.8 & 9.5 \\
\hline Slice $2\{2,2,2,2\}$ & 1.0 & 1.6 & 0.7 & 9.9 \\
\hline Slice $3\{2,2,2,2\}$ & 1.2 & 1.8 & 1.0 & 10.9 \\
\hline Total $\quad\{2,2,2,2\}$ & 1.0 & 1.5 & 0.7 & 9.9 \\
\hline Total $\quad\{3,3,2,2\}$ & 0.7 & 1.1 & 0.6 & 9.4 \\
\hline Total $\{4,4,2,2\}$ & 0.5 & 0.7 & 0.4 & 3.8 \\
\hline \multicolumn{5}{|c|}{ Rodded B } \\
\hline & $\mathrm{AVG}$ & RMS & MRE & MAX \\
\hline Slice $1\{2,2,2,2\}$ & 1.0 & 1.6 & 0.8 & 9.8 \\
\hline Slice $2\{2,2,2,2\}$ & 1.1 & 1.7 & 0.9 & 10.7 \\
\hline Slice $3\{2,2,2,2\}$ & 1.5 & 2.1 & 1.4 & 11.2 \\
\hline
\end{tabular}




\begin{tabular}{|cc|c|c|c|c|}
\hline Total & $\{2,2,2,2\}$ & 1.1 & 1.6 & 0.9 & 10.3 \\
\hline Total $\{3,3,2,2\}$ & 0.7 & 1.1 & 0.6 & 9.0 \\
\hline Total & $\{4,4,2,2\}$ & 0.5 & 0.7 & 0.4 & 3.7 \\
\hline
\end{tabular}

\section{REFERENCES}

*Publications as a result of this project.

1. Briesmeister, J.F., 2000. MCNP e A General Monte Carlo N-Particle Transport Code.

Version 4C. Los Alamos National Laboratory. LA-13709-M.

*2. Forget, B., Rahnema, F. (2005) "Improved Monte Carlo Adaptation of the Heterogeneous

Coarse Mesh Transport Method", Monte Carlo 2005 Topical Meeting,

Chattanooga, Tennessee.

3. T. Simeonov, "Release Notes - Helios System Version 1.8," Studsvik Scandpower Report, SSP-03/221, November 26 (2003).

*4. Forget, B., Rahnema, F. (2005) "3-D Heterogeneous Coarse Mesh Transport Method", International Topical Meeting on Mathematics and Computations, Super-computing, Reactor Physics, and Nuclear and Biological Applications, Avignon, France, September 12-15.

5. Forget, B., Ilas, G., Rahnema, F. (2004) "A Heterogeneous Coarse Mesh Transport Solution for a 2-D CANDU-6 Benchmark problem", Sixth International Conference on Simulation Methods in Nuclear Engineering, Montreal, Canada, October 12-15.

*6. Forget, B., Rahnema, F. (2005) "New Eigenvalue Evaluation Technique in the Heterogeneous Coarse Mesh Transport Method", TRANSACTIONS of the American Nuclear Society $93,511$.

*7. Douglass, S., Rahnema, F. (2009) "2-Dimensional PWR and BWR Whole Core Benchmark Problems", International Conference on Mathematics, Computational Methods \& Reactor Physics (M\&C 2009) Saratoga Springs, New York, May 3-7, 2009, American Nuclear Society, LaGrange Park, IL (2009).

8. D.J. Kelly, "Depletion of a BWR Lattice Using the RACER Continuous Energy Monte Carlo Code," Proceedings of the International Conference on Mathematics and Computations, Reactor Physics and Environmental Analyses, Portland, Oregon, April 30- May 4, Vol. 2, p. 1011, American Nuclear Society (1995).

9. Lewis, E.E., Palmiotti, G., Taiwo, T.A., Blomquist, R.N., Smith, M.A., Tsoulfanidis, N., 2005. Benchmark Specifications for Deterministic MOX Fuel Assembly Transport Calculations without Spatial Homogenization (3-D Extension C5G7 MOX). Nuclear Energy Agency. 
*10. B. Forget and F. Rahnema, "COMET Solutions to Whole Core CANDU-6 Benchmark Problem," PHYSOR-2006: Advances in Nuclear Analysis and Simulation, American Nuclear Society's Topical Meeting on Reactor Physics, Vancouver, BC, Canada, September 10-14 (2006).

*11. Douglass, S. and Rahnema, F. "Whole-Core 1D, 2D, and 3D PWR Benchmark Descriptions", Annals of Nuclear Energy (to be submitted 12/2009).

*12. Douglass, S. and Rahnema, F. "Whole-Core 1D, 2D, and 3D BWR Benchmark Descriptions", Annals of Nuclear Energy (to be submitted 12/2009).

*13. Forget, B., Rahnema, F. "COMET Solution in a Highly Heterogeneous Boiling Water Reactor Benchmark Problem"

*14. Forget, B. "A Three Dimensional Heterogeneous Coarse-Mesh Transport Method for Reactor Calculations" Ph.D. Thesis. 2006.

*15. B. Forget and F. Rahnema, " Comet Solutions to the 3-D C5G7 MOX Benchmark Problem", Progress in Nuclear Energy July 2006: (Volume 48, Issue 5) p. 467

*16. Douglass, S. and Rahnema, F. "Coarse Mesh Solutions to 3D BWR and PWR Benchmark Problems", Annals of Nuclear Energy (to be submitted 1/2009). 